This item was submitted to Loughborough's Research Repository by the author.

Items in Figshare are protected by copyright, with all rights reserved, unless otherwise indicated.

\title{
Reflecting tidal wave beams and local generation of solitary waves in the ocean thermocline
}

PLEASE CITE THE PUBLISHED VERSION

LICENCE

CC BY-NC-ND 4.0

\section{REPOSITORY RECORD}

Akylas, T.R., Roger H.J. Grimshaw, Simon R. Clarke, and A. Tabaei. 2019. "Reflecting Tidal Wave Beams and Local Generation of Solitary Waves in the Ocean Thermocline". figshare. https://hdl.handle.net/2134/2564. 
This item was submitted to Loughborough's Institutional Repository by the author and is made available under the following Creative Commons Licence conditions.



For the full text of this licence, please go to: http://creativecommons.org/licenses/by-nc-nd/2.5/ 


\title{
Reflecting tidal wave beams and local generation of solitary waves in the ocean thermocline
}

\author{
By T. R. A KYLA $S^{1}$, R. H. J. GRIMSHA W $\mathbf{W}^{2}$, \\ S. R. CLARKE ${ }^{3}$ AND ALI TABAEI ${ }^{1}$ \\ ${ }^{1}$ Department of Mechanical Engineering, Massachusetts Institute of Technology, Cambridge, \\ MA 02139, USA \\ ${ }^{2}$ Department of Mathematical Sciences, Loughborough University, Leics LE11 3TU, England \\ ${ }^{3}$ School of Mathematical Sciences, Monash University, Vic. 3800, Australia
}

(Received )

It is generally accepted that ocean internal solitary waves can arise from the interaction of the barotropic tide with the continental shelf, which generates an internal tide that in turn steepens and forms solitary waves as it propagates shorewards. Some field observations, however, reveal large-amplitude internal solitary waves in deep water, hundreds of kilometers away from the continental shelf, suggesting an alternative generation mechanism: tidal flow over steep topography forces a propagating beam of internal tidal wave energy which impacts the thermocline at a considerable distance from the forcing site and gives rise to internal solitary waves there. Motivated by this possibility, a simple nonlinear long-wave model is proposed for the interaction of a tidal wave beam with the thermocline and the ensuing local generation of solitary waves. The thermocline is modelled as a density jump across the interface of a shallow homogeneous fluid layer on top of a deep uniformly stratified fluid, and a finite-amplitude propagating internal wave beam of tidal frequency in the lower fluid is assumed to be incident and reflected at the interface. The induced weakly nonlinear long-wave disturbance on the interface is governed in the far field by an integral-differential equation which accounts for nonlinear and dispersive effects as well as energy loss owing to radiation into the lower fluid. Depending on the intensity of the incident beam, nonlinear wave steepening can overcome radiation damping so a series of solitary waves may arise in the thermocline. Sample numerical solutions of the governing evolution equation suggest that this mechanism is quite robust for typical oceanic conditions.

\section{Introduction}

Internal solitary waves are commonly observed in the coastal oceans and are often modelled by evolution equation of the Korteweg-de Vries type, appropriate for shallow waters (see, for instance, Grimshaw 2001 and Helfrich \& Melville 2006). In coastal regions it is generally accepted that these can be generated through the interaction of 
the barotropic tide with the continental shelf, generating an internal tide that in turn steepens and forms internal solitary waves as it propagates shorewards.

However, based on field observations in the Bay of Biscay, New \& Pingree (1990) first suggested that ocean internal solitary waves may instead arise from the interaction of a propagating internal tidal beam with the thermocline. According to this scenario, the internal tidal beam is itself generated by tidal flow over steep topography near the shelf break, but impacts the thermocline at a considerable distance from the forcing site and thereby generates internal solitary waves propagating offshore in deep water. Their field data, in particular, revealed large-amplitude solitary waves about $150 \mathrm{~km}$ away from the shelf break, making it rather unlikely for these waves to have travelled this far along the thermocline without experiencing significant dissipation. Instead, New \& Pingree $(1990,1992)$ argued that these waves were generated locally by a propagating beam of internal tidal energy which had originated at the topography and, after reflecting from the bottom, encountered the thermocline close to the position where the solitary waves were found. While the original observations had focused on the northern and central parts of the Bay of Biscay, recently Azevedo, da Silva \& New (2006) presented evidence from satellite images that the southern part of this bay is also a site with high-level internal solitary-wave activity, and the same local generation mechanism is applicable there as well.

Gerkema (2001) studied theoretically the local generation of solitary waves by internal tides in a two-layer flow configuration, representing the thermocline as a density jump across the interface of a relatively shallow homogeneous fluid layer on top of a finite-depth stratified fluid. The bottom topography had a smooth shelf profile, and a time-harmonic current of tidal frequency was used as forcing. Gerkema (2001) computed the linear hydrostatic response as an expansion in terms of the long-wave modes of this two-fluid 
system, and argued that local generation of solitary waves is the result of a two-stage process: an internal tidal beam originating at the topography first hits the thermocline and, via an essentially linear mechanism, excites an appreciable long-wave disturbance there, only if the thermocline is moderately strong - a condition that apparently was met in the Bay of Biscay according to the field data. Then, in the course of propagating along the thermocline, the disturbance triggered by the beam experiences the effects of dispersion and nonlinearity, thereby forming solitary waves. This scenario is supported by numerical simulations using a coupled equation system that allows for dispersive and nonlinear effects in the modal expansion of the response; the solitary waves gradually decay though, owing to leakage of energy in the lower fluid as they propagate along the thermocline.

In the present study, the thermocline again is modelled as a density jump across the interface of a homogeneous layer lying on top of a stratified fluid. However, attention is focused on the reflection of a tidal beam that is incident at the interface from the lower fluid, assumed to be infinitely deep, as well as on the evolution of the resulting interfacial disturbance, ignoring the process by which the beam is generated. This simplified approach accounts for the essential ingredients of the mechanism responsible for the local generation of solitary waves, while avoiding a modal expansion.

Internal wave beams derive from the anisotropic nature of wave propagation in stratified fluids, since gravity provides a preferred direction. Ocean internal wave beams, in particular, are excited by tidal flow over bottom topography as demonstrated by theoretical models (Bell 1975, Khatiwala 2003, Lamb 2004) as well as field measurements. In the Bay of Biscay, for instance, Pingree \& New (1989) report observations of a tidal wave beam with amplitudes ranging from approximately $150 \mathrm{~m}$ near the shelf break to $100 \mathrm{~m}$ further out into the deep ocean. 
In the two-fluid configuration considered here, apart from internal waves propagating in the lower fluid with frequencies less than the buoyancy (Brunt-Väisälä) frequency, it is also possible to have interfacial waves; these propagate without leaking energy into the lower fluid, however, only for frequencies above the Brunt-Väisälä frequency. As a result, ignoring nonlinear effects, interfacial disturbances owing to a tidal wave beam, that is incident and reflected at the interface, would be attenuated far from the region of forcing. This suggests that solitary waves could arise from the interaction of a beam with the thermocline only if radiation damping is relatively light, thus allowing nonlinear steepening to come into play.

Assuming that interfacial disturbances are long relative to the depth of the upper fluid layer, a condition that is met for the upper well-mixed layer of the ocean, we find that light radiation damping results under flow conditions analogous to those of a moderately strong thermocline defined by Gerkema (2001). In this flow regime, nonlinear-steepening and dispersive effects can be brought into balance with radiation damping, and the far-field evolution of the interfacial disturbance induced by a finite-amplitude incident and reflected wave beam is governed by an integral-differential equation of the type derived in Maslowe \& Redekopp (1980). Based on numerical solutions of this evolution equation, subject to forcing from the incident and reflected wave beam, it is concluded that nonlinear steepening can overcome radiation damping and solitary waves may form under typical oceanic conditions, supporting the local generation mechanism suggested by New \& Pingree (1990, 1992) and Gerkema (2001).

It appears that the nonlinear long-wave model proposed here could be generalized to account for the Earth's rotation, which is expected to modify the propagation direction of tidal wave beams and also to contribute to the attenuation of solitary waves via the 


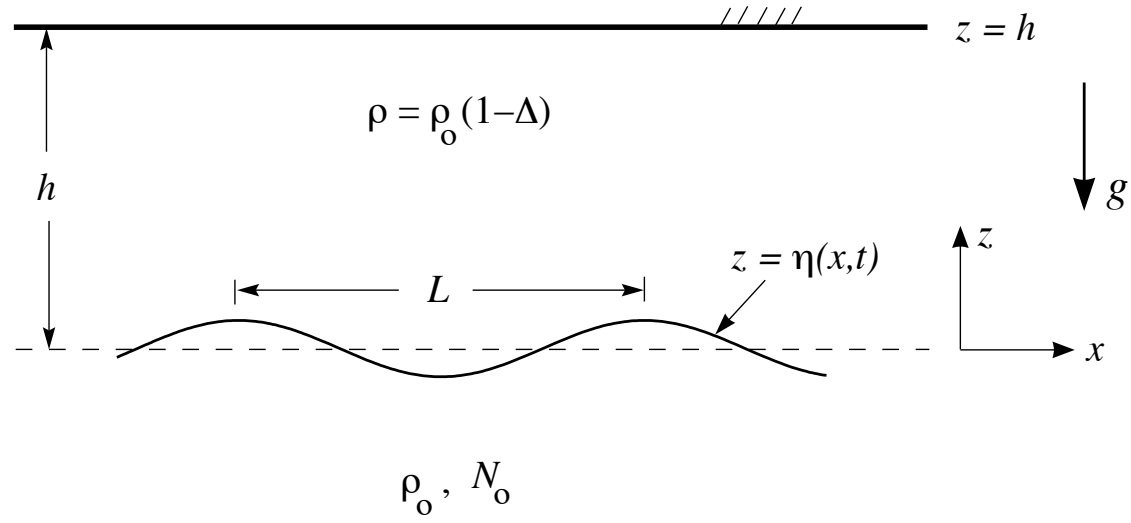

FiguRE 1. Sketch of the two-fluid configuration. The upper fluid layer is homogeneous with density $\rho=\rho_{0}(1-\Delta)$. The lower fluid is uniformly stratified and Boussinesq with reference density $\rho_{0}$ and constant buoyancy frequency $N_{0}$.

generation of dispersive tails (Grimshaw et al. 1998). These effects are not likely to alter the local generation mechanism of solitary waves in an essential way, however.

\section{Flow model and linear response}

Our analysis is based on an inviscid two-fluid configuration sketched in figure 1. A homogeneous fluid of depth $h(0<z<h)$, representing the upper well-mixed layer of the ocean, is bounded by a rigid lid and lies on top of an infinitely deep fluid $(-\infty<z<0)$ which, for simplicity, is taken to be uniformly stratified with (constant) Brunt-Väisälä frequency $N_{0}$, and the Boussinesq approximation is made. Denoting the reference density in the lower fluid by $\rho_{0}$ and the constant density of the upper layer by $\rho_{0}(1-\Delta)$, the density jump $\rho_{0} \Delta$ across the interface $z=0$ represents the thermocline.

This two-fluid system supports interfacial gravity waves owing to the density jump 
at $z=0$ as well as internal gravity waves owing to the buoyancy force in the lower fluid; the former are akin to trapped waves propagating along the ocean thermocline, while the latter can form wave beams similar to those induced by tidal flow over bottom topography. Our interest centres on the possible generation of interfacial solitary waves by an internal wave beam that is incident and reflected at the interface. To address this issue, we shall study the reflection of a finite-amplitude beam at the interface and the subsequent evolution of the resulting interfacial disturbance.

We begin by summarizing the salient features of the linear response; while nonlinear wave steepening is necessary for the formation of solitary waves, the linear response provides motivation for the appropriate scaling of the nonlinear problem in $\S 3$. In dimensionless variables, using $h$ as length scale and $h / c_{0}$ as time scale, $c_{0}=(g h \Delta /(1-\Delta))^{1 / 2}$ being the long-interfacial-wave speed, the governing equations for linear disturbances consist of Laplace's equation

$$
\phi_{x x}+\phi_{z z}=0 \quad(0<z<1)
$$

for the velocity potential $\phi(x, z, t)$ in the upper fluid layer, and

$$
\left(\zeta_{x x}+\zeta_{z z}\right)_{t t}+\mu^{2} \zeta_{x x}=0 \quad(-\infty<z<0)
$$

for the vertical particle displacement $\zeta(x, z, t)$ in the lower fluid, where

$$
\mu=\frac{N_{0} h}{c_{0}} .
$$

These equations are subject to the boundary condition

$$
\phi_{z}=0 \quad(z=1)
$$

at the rigid lid and the following interfacial conditions

$$
\begin{gathered}
\eta_{t}=\phi_{z}=\zeta_{t} \quad(z=0), \\
\eta_{x x}-\phi_{t x x}-\frac{1}{1-\Delta} \zeta_{z t t}=0 \quad(z=0) .
\end{gathered}
$$


It is clear from (2.2) that harmonic plane waves, $\zeta=f(z) \exp \{\mathrm{i}(k x-\omega t)\}$, propagating along $x$ but confined close to the interface, $f(z) \rightarrow 0$ as $z \rightarrow-\infty$, can be found only if

$$
\omega>\mu \text {. }
$$

This condition is also brought out by the dispersion relation of interfacial waves,

$$
\omega^{2}=\frac{k \tanh k}{1+\frac{\left(1-\mu^{2} / \omega^{2}\right)^{1 / 2}}{1-\Delta} \tanh |k|}
$$

that can be readily obtained from the equation system (2.1)-(2.6), taking $\zeta \rightarrow 0$ as $z \rightarrow-\infty$. According to (2.8), $\mu$ is a cut-off frequency below which $k$ is complex, implying that interfacial waves are evanescent rather than propagating along $x$ when $\omega<\mu$.

According to the field observations (New \& Pingree 1990), interfacial disturbances are long compared to $h$ so $k \ll 1$; in this limit, (2.8) can be approximated as

$$
\omega^{2}=k^{2}\left\{1-\frac{\left(1-\mu^{2} / \omega^{2}\right)^{1 / 2}}{1-\Delta}|k|+\ldots\right\} .
$$

Note that, since $\omega^{2} \sim k^{2} \ll 1, \omega^{2} \ll \mu^{2}$ in general; long interfacial waves, therefore, are strongly evanescent unless $\mu \sim k$ or, in terms of the disturbance horizontal length scale $L$,

$$
\mu=O(h / L) \quad \Rightarrow \quad N=\frac{N_{0} L}{c_{0}}=O(1) .
$$

The condition $N=O(1)$, under which long waves can propagate along the interface, may be interpreted as the interfacial-wave time scale $L / c_{0}$ being comparable to $1 / N_{0}$, the time scale of internal waves propagating in the lower fluid. In this sense, (2.10) is analogous to the condition of a moderate-strength thermocline suggested by Gerkema (2001) on the basis of his linear hydrostatic model.

Consider next a propagating harmonic plane internal wave in the lower fluid incident on the interface at an angle $\theta$ to the horizontal (figure 2). The appropriate expressions for $\zeta, \eta$ and $\phi$ are 


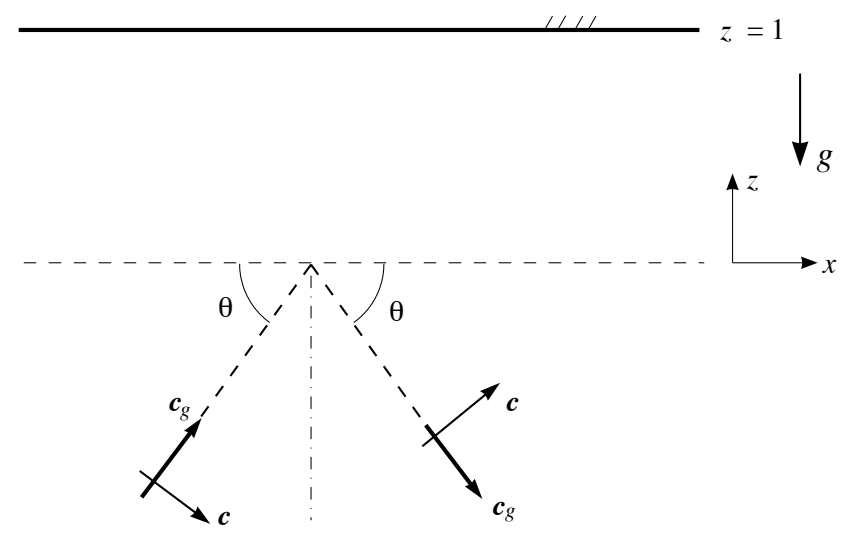

FiguRE 2. A propagating harmonic plane internal wave incident from the lower fluid on the interface at an angle $\theta$ to the horizontal and reflected there. The group velocity $\boldsymbol{c}_{g}$ of the incoming wave points towards the interface, while that of the reflected wave points in the opposite direction. The phase speed $\boldsymbol{c}$ is perpendicular to $\boldsymbol{c}_{g}$.

$$
\begin{gathered}
\zeta=\exp \{\mathrm{i}(k x-l z-\omega t)\}+B \exp \{\mathrm{i}(k x+l z-\omega t)\}, \\
\eta=A \exp \{\mathrm{i}(k x-\omega t)\} \\
\phi=D \cosh k(z-1) \exp \{\mathrm{i}(k x-\omega t)\}
\end{gathered}
$$

where $k, l>0$ and $\omega=\mu \sin \theta=\mu k /\left(k^{2}+l^{2}\right)^{1 / 2}$ according to the familiar dispersion relation of internal waves. Note that, in keeping with causality, the wavevectors in (2.11) have been chosen such that the group velocity (and hence the direction of energy transport) associated with the incoming wave points towards the interface, while that of the reflected wave points in the opposite direction.

The undetermined coefficients $A, B$ and $D$ in $(2.11)-(2.13)$ are found by imposing the interfacial conditions (2.5) and (2.6). The reflection coefficient, in particular, satisfies $|B|^{2}=1$ and is consistent with the expression of Delisi \& Orlanski (1975) in the shortwave limit $(k \gg 1)$. Of more interest here is $A$, the amplitude of the induced interfacial 
wave, which in the long-wave limit $(k \ll 1)$ can be approximated as

$$
A=-\mathrm{i} \frac{\sin 2 \theta}{1-\Delta} \frac{k \mu^{2}}{k^{2}-\mu^{2} \sin ^{2} \theta-\frac{\mathrm{i}}{2} k \mu^{2} \sin 2 \theta /(1-\Delta)} .
$$

As expected, when $\mu \ll 1$ so condition (2.10) is satisfied, the poles of $A$ are close to the real $k$-axis, corresponding to slightly evanescent interfacial waves.

The interfacial disturbance induced by the reflection of an internal wave beam, incident on the interface at an angle $\theta$ to the horizontal, can be readily found by superposition. Specifically, an incoming beam with frequency $\omega=\mu \sin \theta$ takes the form

$$
\zeta_{\text {inc }}=\frac{\mathrm{e}^{-\mathrm{i} \omega t}}{2 \pi} \int_{0}^{\infty} Q(k) \exp \{\mathrm{i} k(x-z \cot \theta)\} \mathrm{d} k,
$$

where $Q(k)$ is related to the specific beam profile and $k$ is restricted to $k>0$, so that energy is transported towards the interface in accordance with figure 2. The induced interfacial disturbance then is

$$
\eta=\frac{e^{\mathrm{i} \omega t}}{2 \pi} \int_{0}^{\infty} Q(k) A(k) e^{\mathrm{i} k x} \mathrm{~d} k
$$

where $A(k)$ is the known interfacial amplitude for plane-wave reflection, given by $(2.14)$ in the long-wave limit.

Since the poles of $A(k)$ are away from the real $k$-axis, the linear response (2.16) is locally confined, $\eta \rightarrow 0$ as $x \rightarrow \pm \infty$. However, as remarked earlier, in the case $\mu \ll 1, A$ has a pole at $k=k_{*}$ slightly above the positive $k$-axis:

$$
k_{*}=\omega+\mathrm{i} \frac{\omega \mu \cos \theta}{2(1-\Delta)},
$$

which provides the far-field response for $x \rightarrow \infty$ :

$$
\eta \sim \frac{\mu^{2} \sin 2 \theta}{2(1-\Delta)} Q(\omega) \exp \left(-\frac{\omega \mu \cos \theta}{2(1-\Delta)} x\right) \mathrm{e}^{\mathrm{i} \omega(x-t)} \quad(x \rightarrow \infty) .
$$

According to (2.18), under flow conditions for which $\mu \ll 1$, the far-field response at the interface propagates to the right of the incident beam only, and is in the form of a long sinusoidal wave with slowly decaying amplitude owing to leaking of energy into 
the lower fluid. A similar interfacial response was predicted by the linear hydrostatic model of Gerkema (2001) for a thermocline of moderate strength and, moreover, solitary waves were seen to form when nonlinear and dispersive effects were included under this flow condition. As remarked earlier, the condition of moderate-strength thermocline in Gerkema (2001) is analogous to $\mu \sim h / L \ll 1$ in the present model, and the possibility that nonlinear steepening may overcome the radiation damping of the linear response (2.18) to give rise to solitary waves is examined below.

\section{Nonlinear response}

\subsection{Reflection of tidal wave beam}

Based on the linear analysis of $\S 2$, long interfacial waves of wavenumber $k$ and frequency $\omega$ are subject to light radiation damping, so nonlinear steepening is likely to be more effective, when $k \sim \omega \sim \mu \ll 1$. This suggests treating $\mu$ as a small parameter and re-scaling $x$ and $t$ as follows:

$$
X=\mu x, \quad T=\mu t \quad(\mu \ll 1) .
$$

Furthermore, from (2.18), such lightly damped interfacial waves are triggered by wave beams incident on the interface from below at an angle $\theta$ to the horizontal, where $\theta=O(1)$ in general. In the lower fluid, therefore, the vertical coordinate is scaled in sympathy with $X$ :

$$
Z=\mu z
$$

The vertical particle displacement $\zeta(X, Z, T)$ then is governed, to leading order in $\mu$, by a linear equation analogous to $(2.2)$ :

$$
\left(\zeta_{X X}+\zeta_{Z Z}\right)_{T T}+\zeta_{X X}=0 \quad(-\infty<z<\eta),
$$

even for finite-amplitude disturbances.

Turning next to the interfacial elevation $\eta(X, T)$, according to (2.14), for long waves 
with $k=O(\mu)$, the ratio of the interfacial amplitude to the incident-wave amplitude is $O(\mu)$. This suggests that the interfacial disturbance induced by a finite-amplitude long-wave beam is weakly nonlinear, namely

$$
\eta=\mu \tilde{\eta}
$$

as a result, to leading order in $\mu$, the linearized interfacial conditions (2.5) and (2.6) on $z=0$ continue to hold:

$$
\begin{gathered}
\phi_{z}=\mu \zeta_{T}=\mu^{2} \tilde{\eta}_{T} \quad(z=0), \\
\tilde{\eta}_{X X}-\phi_{T X X}-\frac{1}{1-\Delta} \zeta_{Z T T}=0 \quad(z=0) .
\end{gathered}
$$

Furthermore, the velocity potential in the upper fluid layer, $\phi(z ; X, T)$, which satisfies Laplace's equation (2.1) and the rigid-lid condition (2.4), can be expanded as

$$
\phi=a(X, T)-\frac{1}{2} \mu^{2}(z-1)^{2} a_{X X}+\cdots .
$$

Therefore, according to (3.5), $\tilde{\eta}_{T}=a_{X X}=\phi_{X X}$ so (3.6) becomes a forced wave equation for $\tilde{\eta}(X, T)$ :

$$
\tilde{\eta}_{X X}-\tilde{\eta}_{T T}=\left.\frac{1}{1-\Delta} \zeta_{Z T T}\right|_{Z=0}
$$

The forcing on the right-hand side of (3.8) derives from the incident and reflected internal wave beam at the interface. Specifically, for a finite-amplitude beam incident at an angle $\theta$ to the horizontal with frequency $\Omega=\sin \theta$, it follows from equation (3.3) that

$$
\zeta=\{q(X-Z \cot \theta)+r(X+Z \cot \theta)\} \mathrm{e}^{-\mathrm{i} \Omega T}+\text { c.c. },
$$

where c.c. stands for the complex conjugate. However, according to $(3.5), \zeta=O(\mu)$ on $Z=0$, implying that the incident beam is nearly perfectly reflected at the interface, so $r(X)=-q(X)$ to leading order; hence,

$$
\left.\zeta_{Z T T}\right|_{Z=0}=2 \Omega^{2} \cot \theta q_{X} \mathrm{e}^{-\mathrm{i} \Omega T}+\text { c.c. },
$$


where

$$
q(X)=\frac{1}{2 \pi} \int_{0}^{\infty} \hat{q}(k) \mathrm{e}^{\mathrm{i} k X} \mathrm{~d} k
$$

so, in accordance with the radiation condition noted earlier (see figure 2), the incoming and reflected beam profiles $q(X)$ and $r(X)$ involve plane waves with $k>0$ only.

Finally, inserting (3.10) in (3.8), the interfacial response $\tilde{\eta}(X, T)$ satisfies

$$
\tilde{\eta}_{X X}-\tilde{\eta}_{T T}=\frac{\sin 2 \theta}{1-\Delta} q_{X} \mathrm{e}^{-\mathrm{i} \Omega T}+\text { c.c.. }
$$

This forced wave equation can be readily solved by taking Fourier transform in $X$. The response propagates to the right of the incident beam $(X>0)$ only and, assuming that the forcing owing to the incident and reflected beam is turned on at $T=0$, is given by

$$
\tilde{\eta}(X, T) \sim \frac{\sin 2 \theta}{2(1-\Delta)} \hat{q}(\Omega) \mathrm{e}^{\mathrm{i} \Omega(X-T)} \mathrm{H}(T-X)+\text { c.c. } \quad(X \rightarrow \infty),
$$

where $\mathrm{H}(X)$ stands for the Heaviside function.

According to (3.13), to leading order in $\mu$, the reflection of a finite-amplitude beam at the interface gives rise to a weakly nonlinear sinusoidal long wave there, which propagates to the right of the incident beam. Upon comparison with the linear response (2.18), radiation damping is not present in (3.13); it comes into play for $X=O(1 / \mu)$, along with the nonlinear and dispersive effects as it turns out. A far-field analysis that takes into account these effects is presented below.

\subsection{Far-field evolution}

For the purpose of analyzing the far-field response, we shall adopt a reference frame moving with the interfacial linear-long-wave speed, $\Theta=X-T$, and introduce $\xi=$ $\mu X=\mu^{2} x$ so that $\xi=O(1)$ in the far field. In terms of these variables, the near-field response (3.13) may be viewed as an 'inner' solution valid as $\xi \rightarrow 0$, and the far-field response $\eta(\Theta, \xi)(-\infty<\Theta<\infty, \xi>0)$, which is the 'outer' solution, obeys the matching 
condition:

$$
\tilde{\eta} \rightarrow \frac{\sin 2 \theta}{2(1-\Delta)} \hat{q}(\Omega) \mathrm{e}^{\mathrm{i} \Omega \Theta} \mathrm{H}(-\Theta)+\text { c.c. } \quad(\xi \rightarrow 0) .
$$

As the incident and reflected beam is not present in the far field, $\tilde{\eta}(\Theta, \xi)$ thus propagates as a free disturbance for $\xi>0$, and (3.14) provides the necessary connection with the forcing owing to these beams.

We now derive an equation that governs the evolution of the far-field response for $\xi>0$. Consider the lower fluid first. Since no forcing is present, the far-field vertical particle displacement accompanying $\tilde{\eta}(\Theta, \xi)$ is weakly nonlinear, $\zeta=\mu \tilde{\zeta}(\Theta, Z, \xi)$ and, from (3.3), satisfies the linear equation

$$
\tilde{\zeta}_{\Theta \Theta}+\tilde{\zeta}_{Z Z}+\tilde{\zeta}=0 \quad(-\infty<Z<0),
$$

to leading order in $\mu$.

Turning next to the upper fluid layer, using Laplace's equation (2.1) along with the rigid-lid condition $(2.4)$, the velocity potential $\phi(z ; \Theta, \xi)$ has an expansion analogous to (3.7):

$$
\phi=\tilde{a}(\Theta, \xi)-\frac{1}{2} \mu^{2}(z-1)^{2} \tilde{a}_{\Theta \Theta}-\mu^{3}(z-1)^{2} \tilde{a}_{\Theta \xi}+\cdots .
$$

Imposing the kinematic conditions at the interface $z=\mu \tilde{\eta}$ and making use of (3.16) then yields

$$
\begin{gathered}
\tilde{a}_{\Theta}=-\tilde{\eta}-\mu \tilde{\eta}^{2}-2 \mu \tilde{a}_{\xi}+O\left(\mu^{2}\right), \\
\left.\tilde{\zeta}\right|_{Z=0}=\tilde{\eta}+O\left(\mu^{2}\right) .
\end{gathered}
$$

Finally, the dynamic condition requires the pressure to be continuous at the interface $z=\mu \tilde{\eta}$. For our two-fluid system, Thorpe (1998) has derived an approximation to this condition, correct to third order in the amplitude, that can be applied at $z=0$. 
Implementing the long-wave scalings valid here, this leads to

$$
\left(\tilde{\eta}+\tilde{a}_{\Theta}\right)_{\Theta}+\mu\left(\tilde{\eta}_{\xi}+\tilde{a}_{\Theta \xi}-\frac{1}{2}\left(\tilde{a}_{\Theta}^{2}\right)_{\Theta}-\left.\frac{1}{1-\Delta} \tilde{\zeta}_{\Theta Z}\right|_{Z=0}\right)=O\left(\mu^{2}\right) .
$$

Using then (3.17) to eliminate $\tilde{a}$ from (3.19) results in the following evolution equation for $\tilde{\eta}(\Theta, \xi)$ :

$$
2 \tilde{\eta}_{\xi}-3 \tilde{\eta} \tilde{\eta}_{\Theta}-\frac{1}{1-\Delta}\left(\left.\tilde{\zeta}_{Z}\right|_{Z=0}\right)_{\Theta}=0 .
$$

The third term in (3.20), which accounts for the presence of the lower fluid, can be expressed in terms of $\tilde{\eta}$ by solving equation (3.15) subject to the boundary condition (3.18) on $Z=0$ and the appropriate radiation condition as $Z \rightarrow-\infty$. Specifically, taking Fourier transform in $\Theta$,

$$
\hat{\eta}(\kappa ; \xi)=\int_{-\infty}^{\infty} \mathrm{e}^{-\mathrm{i} \kappa \Theta} \tilde{\eta}(\Theta, \xi) \mathrm{d} \Theta
$$

one has

$$
\left.\tilde{\zeta}_{Z}\right|_{Z=0}=\frac{1}{2 \pi} \int_{-\infty}^{\infty} \mathrm{i} m \mathrm{e}^{\mathrm{i} \kappa \Theta} \hat{\eta}(\kappa ; \xi) \mathrm{d} \kappa
$$

where

$$
m= \begin{cases}-\mathrm{i}\left(\kappa^{2}-1\right)^{\frac{1}{2}} & \left(\kappa^{2}>1\right) \\ \operatorname{sgn} \kappa\left(1-\kappa^{2}\right)^{\frac{1}{2}} & \left(\kappa^{2}<1\right) .\end{cases}
$$

The choice of the branch of the square root in (3.23) is dictated by causality; it ensures that, as $Z \rightarrow-\infty$, the disturbance in the lower fluid either decays (for $\kappa^{2}>1$ ) or transports energy away from the interface (for $\kappa^{2}<1$ ), resulting in radiation damping of $\tilde{\eta}$.

Combining (3.20) with (3.22), the evolution equation governing the far-field response $\tilde{\eta}(\Theta, \xi)$

$$
2 \tilde{\eta}_{\xi}-3 \tilde{\eta} \tilde{\eta}_{\Theta}+\frac{1}{1-\Delta} \frac{1}{2 \pi} \int_{-\infty}^{\infty} \kappa m \mathrm{e}^{\mathrm{i} \kappa \Theta} \hat{\eta}(\kappa ; \xi) \mathrm{d} \kappa=0,
$$

is an integral-differential equation of the type derived earlier by Maslowe \& Redekopp (1980) for the purpose of discussing the radiation damping of weakly nonlinear long waves 
in stratified shear flows of large depth. If the lower fluid is not stratified so radiation damping is absent, (3.23) is replaced by $m=-\mathrm{i}|\kappa|$ and (3.24) reduces to the familiar Benjamin-Davis-Ono (BDO) equation for the propagation of long interfacial waves in deep fluids. As is well known, the BDO equation admits solitary-wave solutions with algebraic profiles.

In the present context, equation (3.24) is to be solved subject to the 'initial' condition (3.14) at $\xi=0$ that ensures matching with the near-field response. Ignoring nonlinearity, the far-field response eventually decays via radiation damping as implied by (2.18), and the question of interest here is whether nonlinear steepening, represented by the second term in (3.24), can reverse this trend so that the disturbance induced by the tidal beam can give rise to solitary waves. This issue is now taken up by solving equation (3.24) subject to (3.14) numerically.

\section{Numerical results}

In our computations, the incident tidal beam is taken to have a profile of the form

$$
\zeta_{\text {inc }}=\frac{C}{2} \exp (-|\chi| / \alpha) \mathrm{e}^{-\mathrm{i} \Omega T}+\text { c.c. },
$$

where $\chi$ denotes the cross-beam coordinate, and the parameters $C$ and $\alpha$ control the peak amplitude and the width of the wave beam, respectively. For a beam incident on the interface at an angle $\theta$ to the horizontal, $\chi=X \sin \theta-Z \cos \theta$, and (4.1) leads to the following expression for $q$ in (3.9)

$$
q(X)=\frac{C}{2} \exp \left(-\frac{\Omega}{\alpha}|X|\right),
$$

where $\Omega=\sin \theta$; its Fourier transform is given by

$$
\hat{q}(k)=\frac{\alpha C}{\Omega\left(1+\left(\alpha^{2} k^{2}\right) / \Omega^{2}\right)},
$$


SO

$$
\hat{q}(\Omega)=\frac{\alpha C}{\Omega\left(1+\alpha^{2}\right)}
$$

The evolution equation (3.24) for $\tilde{\eta}(\Theta, \xi)$ is solved numerically as an initial-value problem, treating the matching condition (3.14) as initial condition at $\xi=0$ and advancing $\tilde{\eta}$ in $\xi>0$. For this purpose, we employ a pseudospectral technique in which, at each step $\Delta \xi$, the nonlinear term in (3.24) is advanced by a forward Euler finite-difference scheme while the linear term is treated by Fourier transform using the fast Fourier transform in a finite computational domain $\Theta_{-\infty}<\Theta<\Theta_{+\infty}$.

Inserting (4.4) in (3.14), the initial condition applied at $\xi=0$ is

$$
\tilde{\eta}(\Theta, \xi=0)=\frac{2 \alpha C \cos \theta}{(1-\Delta)\left(1+\alpha^{2}\right)} \sin (\Omega \Theta) \mathrm{H}(-\Theta),
$$

where the phase of the incident wave beam has been chosen so as to avoid a discontinuity at $\Theta=0$. Moreover, in implementing (4.5) numerically, $\tilde{\eta}(\Theta, \xi=0)$ was tapered off as $\Theta \rightarrow-\infty$ so that the disturbance goes to zero and does not reach the boundaries of the computational domain. Note that while the parameter $\mu$ does not affect $\tilde{\eta}(\Theta, \xi)$, it plays an important part in transforming the spatial evolution $\tilde{\eta}(\Theta, \xi)$ to the temporal evolution $\tilde{\eta}(X, T)$, as well as in determining the interface elevation, $\eta=\mu \tilde{\eta}$.

Figure 3 displays results at different values of $\xi$ from a sample run using a computational domain $\Theta_{+\infty}-\Theta_{+\infty}=1536$ with 8192 grid points along $\Theta$ and step size $\Delta \xi=0.0001$, for the choice of parameters $\theta=10^{\circ}, C=1.5$ and $\alpha=1.0$. Each station $\xi$ specifies a fixed location along the interface, and $\tilde{\eta}(\Theta, \xi)$ furnishes the corresponding time history of the response there. The sinusoidal disturbance triggered by the beam initially steepens in a typical hyperbolic fashion owing to the nonlinear term in (3.24) and, as dispersive effects become important, trains of solitary waves are seen to emerge, 


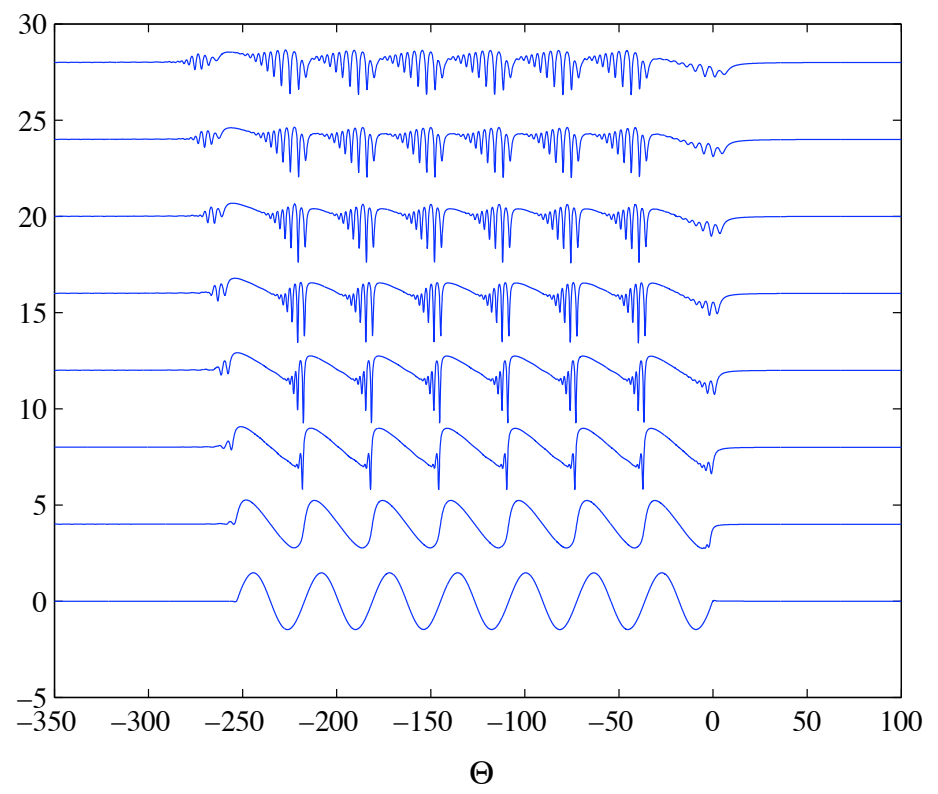

Figure 3. The far-field response $\tilde{\eta}(\Theta, \xi)$ at various stations $\xi$ along the interface for the choice of parameters $\theta=10^{\circ}, C=1.5$ and $\alpha=1.0$. The bottom plot is the 'initial' condition (4.5) at $\xi=0$, and $\xi$ increases upwards by steps of 2 .

but eventually radiation damping comes into play, causing the solitary waves to decay far from the region of forcing.

Next we apply our model to the local generation of solitary waves in the ocean thermocline. For the tidal period $\tau=12 \mathrm{hrs}$, the angle $\theta$ at which the incoming beam hits the thermocline is fixed by the dispersion relation of internal waves in the lower fluid, $\sin \theta=\tau /\left(2 \pi N_{0}\right)$. In the ocean, the buoyancy frequency $N_{0}$ is in the range 1$3 \times 10^{-3} \mathrm{sec}^{-1}$, so $\theta$ varies between roughly $3^{0}$ and $8^{0}$. Moreover, taking $h=60 \mathrm{~m}$ and $\Delta=1.5 \times 10^{-3}$ as in Gerkema (2001), it follows from (2.3) that the parameter $\mu$ is in the range $0.06-0.2$. We chose $N_{0}=2 \times 10^{-3} \mathrm{sec}^{-1}$, the value of $N_{0}$ assumed by Gerkema (2001), which implies $\theta=4.17^{0}$ and $\mu=0.128$. We also took $\alpha=0.65$, corresponding to an incoming beam of width roughly $25 \mathrm{~km}$ along the horizontal, as suggested by figure 3 of Gerkema (2001). Finally, we used a computational domain $\Theta_{+\infty}-\Theta_{+\infty}=2048$ with 8192 points along $\Theta$ and step size $\Delta \xi=0.0001$. 

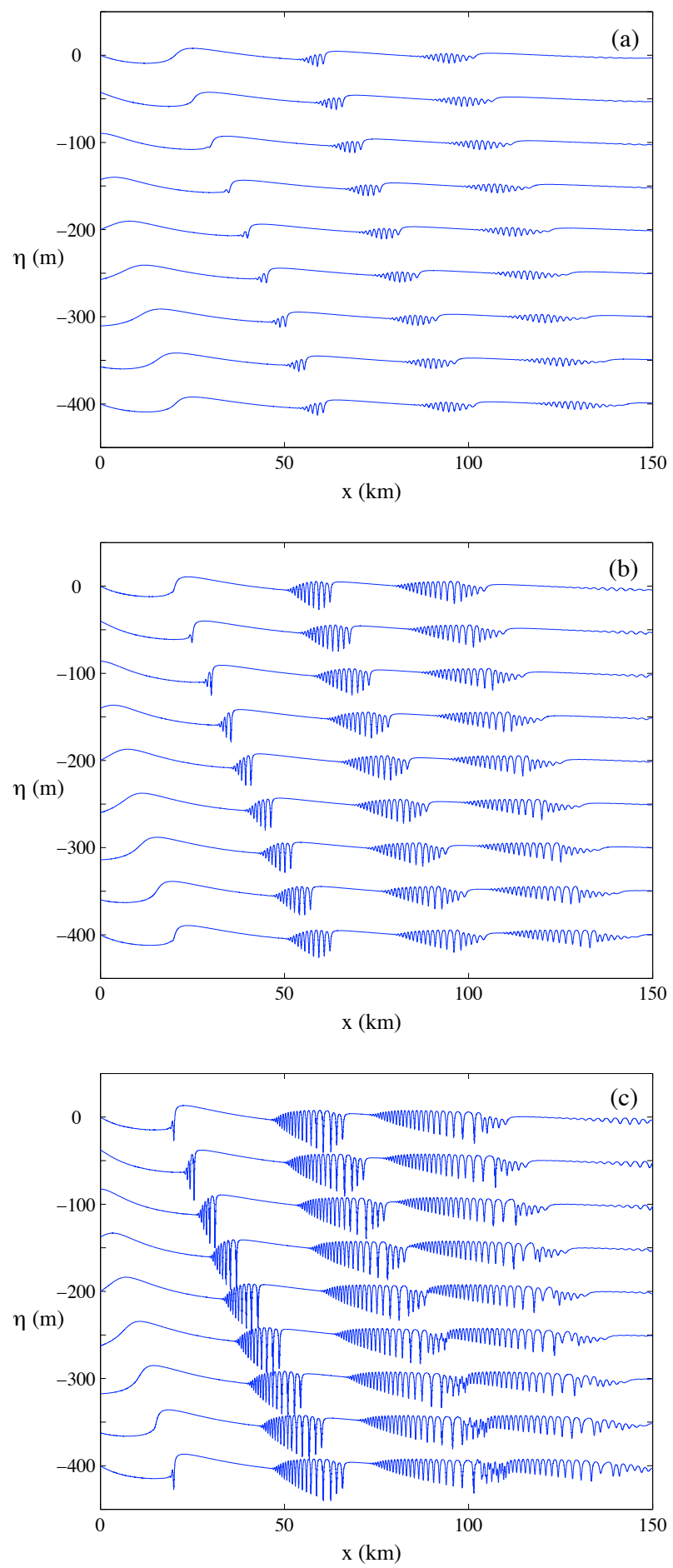

FigURE 4. Snapshots of the far-field interfacial response induced by a tidal beam incident on the thermocline at an angle $\theta=4.17^{\circ}$ to the horizontal, under flow conditions corresponding to $\mu=0.128$. The interface elevation $\eta$ is displayed every $1.5 \mathrm{hrs}$ within a tidal period, $42 \mathrm{hrs}$ after the beam has been turned on (time increases from top to bottom). The beam profile has fixed width, corresponding to $\alpha=0.65$ in (4.1), and the amplitude parameter $C$ is varied: (a) $C=1.5$; (b) $C=2.0$; (c) $C=2.5$. 
Figure 4 shows snapshots every $1.5 \mathrm{hrs}$ within a tidal period of the interface elevation $\eta$ in dimensional variables for three values of the amplitude parameter, $C=1.5, C=2$ and $C=2.5$. These values of $C$ correspond to peak vertical displacement amplitudes of $90 \mathrm{~m}, 120 \mathrm{~m}$ and $150 \mathrm{~m}$ for the incoming beam, which fall in the low, middle and high end, respectively, of the range of observed beam amplitudes reported by Pingree \& New (1989) in the Bay of Biscay. Clearly, the generation mechanism of solitary waves is sensitive to the beam strength; increasing the amplitude of the incoming beam from $90 \mathrm{~m}$ to $150 \mathrm{~m}$ causes the generated solitary waves to become significantly steeper and to occur closer to the region where the beam reflects at the thermocline. While no direct comparison can be made with the simulations in Gerkema (2001), the evolution depicted in figure 4(b) for a beam with peak amplitude $120 \mathrm{~m}$ is qualitatively similar with the evolution of the interface shown in his figure 8 for a moderate-strength thermocline. Note, in particular, that the first solitary wave appears on a trough of the induced disturbance, roughly $35 \mathrm{~km}$ from the position where the beam impacts the thermocline, and attains a peak amplitude of about $25 \mathrm{~m}$ before radiation damping sets in, as seen in the simulation.

The simple theoretical model proposed here adds to the understanding of the mechanism responsible for local generation of solitary waves in the ocean thermocline. Consistent with the two-stage process envisaged by Gerkema (2001), a finite-amplitude tidal beam reflecting at the thermocline first induces a weakly nonlinear disturbance there which, under conditions of light damping, then forms trains of solitary waves owing to the combined action of nonlinearity and dispersion, as it propagates away from the region of forcing. Although results were presented for flow conditions corresponding to those in Gerkema (2001), we also carried out computations for other parameter values typical of oceanic conditions, confirming the qualitative scenario of local generation of solitary waves illustrated in figure 4. Our computations, in particular, reveal that the intensity of 
the incoming beam plays a key part in determining where the solitary waves form as well as their steepness. This suggests that a strong enough incoming beam could still generate solitary waves when the additional dispersive effects owing to the Earth's rotation are taken into account.

This work was initiated while the first author (TRA) was visiting Loughborough University and the third author (SRC) was visiting University College, London. Both wish to acknowledge the support of EPSRC grants which made these visits possible. TRA also acknowledges the Air Force Office of Scientific Research, Air Force Materials Command, USAF, Grant FA9950-04-1-0125 and the National Science Foundation Grant DMS-

0305940.

\section{REFERENCES}

Azevedo, A., DA Silva, J. C. B. \& New, A. L. 2006 On the generation and propagation of internal solitary waves in the southern Bay of Biscay. Deep-Sea Res. 53, 927-941.

BeLL, T. H. 1975 Lee waves in stratified flows with simple harmonic time dependence. J. Fluid Mech. 67, 705-722.

Delisi, D. P. \& ORLAnski, I. 1975 On the role of density jumps in the reflexion and breaking of internal gravity waves. J. Fluid Mech. 69, 445-464.

Gerkema, T. 2001 Internal and interfacial tides: Beam scattering and local generation of solitary waves. J. Mar. Res. 59, 227-255.

Grimshaw, R. 2001 Internal solitary waves. In: Environmental stratified flows (ed. R. Grimshaw). Boston: Kluwer.

Grimshaw, R. H. J., Ostrovsky, L. A., Shrira, V. I. \& Stepanyants, Y. A. 1998 Long nonlinear surface and internal gravity waves in a rotating ocean. Nonlinear Processes in Geophysics 19, 289-338.

Helfrich, K. R. \& Melville, W. K. 2006 Long nonlinear internal waves. Annu. Rev. Fluid Mech. 38, 395-425.

Khatiwala, S. 2003 Generation of internal tides in an ocean of finite depth: Analytical and numerical calculations. Deep-Sea Res. 50, 3-21.

LAmв, K. G. 2004 Nonlinear interaction among internal wave beams generated by tidal flow over supercritical topography. Geophys. Res. Lett. 31, doi: 10.1029/2003GL019393.

Maslowe, S. A. \& Redekopp, L. G. 1980 Long nonlinear waves in stratified shear flows. $J$. Fluid Mech. 101, 321-348.

New, A. L. \& Pingree, R. D. 1990 Large-amplitude internal soliton packets in the central Bay of Biscay. Deep-Sea Res. 37, 513-524.

New, A. L. \& Pingree, R. D. 1992 Local generation of internal soliton packets in the central Bay of Biscay. Deep-Sea Res. 39, 1521-1534.

Pingree, R. D. \& New, A. L. 1989 Downward propagation of internal tidal energy into the Bay of Biscay. Deep-Sea Res. 36, 735-758.

Thorpe, S. A. 1998 Nonlinear reflection of internal waves at a density discontinuity at the base of the mixed layer. J. phys. oceanogr. 28, 1853-1860. 